\title{
OPIAL TYPE DISCRETE INEQUALITIES IN TWO VARIABLES
}

\author{
B. G. PACHPATTE
}

\begin{abstract}
The aim of the present note is to establish two new discrete inequalities of the Opial type involving functions of two variables and their differences. The analysis used in the proofs is elementary and the results established provide new estimates on these types of inequalities.
\end{abstract}

\section{Introduction.}

The discrete inequality

$$
\sum_{i=1}^{n} u_{i}^{p}\left(u_{i}-u_{i-1}\right) \leq \frac{(n+1)^{p}}{p+1} \sum_{i=1}^{n}\left(u_{i}-u_{i-1}\right)^{p+1},
$$

valid for a nondecreasing sequence $\left\{u_{i}\right\}$ of nonnegative real numbers with $u_{0}=0$ and $p \geq$ 1 is established in 1967 by Wong [8]. Inequality (1) is a discrete analogue of the variant of Opial's inequality [5] given by Hua in [2]. Subsequently, many results have appeared in the literature concerning various generalizations and extensions of $(1)$, see $[1,3,4,6,7]$. The main object of the present note is to establish two new discrete inequalities involving functions of two variables which claim their origin to the inequality (1). An interesting feature of the inequalities established here is that the analysis used in their proofs is elementary and our results provide new estimates on these types of inequalities.

\section{Statement of results.}

We first introduce the basic notations and definitions used in our discussion. Let $N=\{1,2, \cdots\}$ and $N_{0}=\{0,1,2, \cdots, n\}, M_{0}=\{0,1,2, \cdots, m\}$ for $n, m \in N$ and $Q=$ $N_{0} \times M_{0}$. We shall use the usual convention of writing $z(x, y)=0$ if $x \notin N_{0}$ or $y \notin M_{0}$ or both $x \notin N_{0}$ and $y \notin M_{0}$ where $z(x, y)$ is a function defined on $Q$. We also use the following definitions and notations of the operators:

$$
\begin{aligned}
\nabla_{1} z(x, y) & =z(x, y)-z(x-1, y) \\
\nabla_{2} z(x, y) & =z(x, y)-z(x, y-1) \\
\nabla_{2} \nabla_{1} z(x, y) & =\nabla_{1} z(x, y)-\nabla_{1} z(x, y-1) .
\end{aligned}
$$

Received August 8, 1990. 
for $(x, y) \in Q$.

Our main result is given in the following theorem.

Theorem $\mathbb{1}$. Let $u(x, y)$ be a real-valued function defined for $(x, y) \in Q$ for which $\nabla_{1} u(x, y), \nabla_{2} u(x, y), \nabla_{2} \nabla_{1} u(x, y)$ exist for $(x, y) \in Q$ and such that $u(0, y)=0, u(n, y)$ $=0, u(x, 0)=0, u(x, m)=0$ for $x \in N_{0}, y \in M_{0}$ and $1 \leq p_{i}<\infty$ for $i=1,2,3,4$, then

$$
\begin{aligned}
& \sum_{x=1}^{n} \sum_{y=1}^{m}|u(x, y)|^{p_{1}}\left|\nabla_{1} u(x, y)\right|^{p_{2}}\left|\nabla_{2} u(x, y)\right|^{p_{3}}\left|\nabla_{2} \nabla_{1} u(x, y)\right|^{p_{4}} \\
& \leq K \prod_{i=1}^{4}\left\{\sum_{x=1}^{n} \sum_{y=1}^{m}\left|\nabla_{2} \nabla_{1} u(x, y)\right|^{2 p_{i}}\right\}^{\frac{1}{2}},
\end{aligned}
$$

where

$$
K=\left(\frac{1}{2}\right)^{2 p_{1}+p_{2}+p_{3}} n^{p_{1}+p_{3}-1} m^{p_{1}+p_{2}-1}
$$

As a consequence of Theorem 1 , we have the following

Theorem 2. Assume that in the hypotheses of Theorem 1 we have $p_{1}=p_{2}=p_{3}=$ $p_{4}=1$. Then

$$
\begin{aligned}
\sum_{x=1}^{n} \sum_{y=1}^{m} \mid u(x, y) \| \nabla_{1} u(x, y) & \left\|\nabla_{2} u(x, y)\right\| \nabla_{2} \nabla_{1} u(x, y) \mid \\
\leq & K_{0} \sum_{x=1}^{n} \sum_{y=1}^{m}\left|\nabla_{2} \nabla_{1} u(x, y)\right|^{4},
\end{aligned}
$$

where $K_{0}=\left(\frac{n m}{4}\right)^{2}$.

A slight variant of the inequality (2) is embodied in the following theorem.

Theorem 3. Assume that the hypotheses of Theorem 1 hold except that $1 \leq p_{i}<\infty$ for $i=1,2,3$. Then

$$
\begin{aligned}
\sum_{x=1}^{n} \sum_{y=1}^{m}|u(x, y)|^{p_{1}}\left|\nabla_{1} u(x, y)\right|^{p_{2}}\left|\nabla_{2} u(x, y)\right|^{p_{3}} \\
\leq L \prod_{i=1}^{3}\left\{\sum_{x=1}^{n} \sum_{y=1}^{m}\left|\nabla_{2} \nabla_{1} u(x, y)\right|^{p_{i}}\right\},
\end{aligned}
$$

where

$$
L=\left(\frac{1}{2}\right)^{2 p_{1}+p_{2}+p_{3}} n^{p_{1}+p_{3}-2} m^{p_{1}+p_{2}-2} .
$$

As an immediate consequence of Theorem 3, we have the following 
Theorem 4. Assume that in the hypotheses of Theorem 3 we have $p_{1}=p_{2}=p_{3}=1$. Then

$$
\begin{aligned}
\sum_{x=1}^{n} \sum_{y=1}^{m} \mid u(x, y) & \left\|\nabla_{1} u(x, y)\right\| \nabla_{2} u(x, y) \mid \\
\leq & \mathbb{K}_{0} \sum_{x=1}^{n} \sum_{y=1}^{m}\left|\nabla_{2} \nabla_{1} u(x, y)\right|^{3},
\end{aligned}
$$

where $K_{0}$ is as defined in Theorem 2.

3. Proofs of Theorems $1-4$.

From the hypotheses of Theorem 1 , it is easy to observe that the following identities hold:

$$
\begin{aligned}
u(x, y) & =\sum_{s=1}^{x} \sum_{t=1}^{y} \nabla_{2} \nabla_{1} u(s, t), \\
u(x, y) & =-\sum_{s=1}^{x} \sum_{t=y+1}^{m} \nabla_{2} \nabla_{1} u(s, t), \\
u(x, y) & =-\sum_{s=x+1}^{n} \sum_{t=1}^{y} \nabla_{2} \nabla_{1} u(s, t), \\
u(x, y) & =\sum_{s=x+1}^{n} \sum_{t=y+1}^{m} \nabla_{2} \nabla_{1} u(s, t), \\
\nabla_{1} u(x, y) & =\sum_{t=1}^{y} \nabla_{2} \nabla_{1} u(x, t), \\
\nabla_{1} u(x, y) & =-\sum_{t=y+1}^{m} \nabla_{2} \nabla_{1} u(x, t), \\
\nabla_{2} u(x, y) & =\sum_{s=1}^{x} \nabla_{2} \nabla_{1} u(s, y), \\
\nabla_{2} u(x, y) & =-\sum_{s=x+1}^{n} \nabla_{2} \nabla_{1} u(s, y),
\end{aligned}
$$

for $(x, y) \in Q$. From $(6)-(9),(10)-(11),(12)-(13)$ we observe that 


$$
\begin{aligned}
|u(x, y)| & \leq\left(\frac{1}{2}\right)^{2} \sum_{s=1}^{n} \sum_{t=1}^{m}\left|\nabla_{2} \nabla_{1} u(s, t)\right|, \\
\left|\nabla_{1} u(x, y)\right| & \leq \frac{1}{2} \sum_{t=1}^{m}\left|\nabla_{2} \nabla_{1} u(x, t)\right|, \\
\left|\nabla_{2} u(x, y)\right| & \leq \frac{1}{2} \sum_{s=1}^{n}\left|\nabla_{2} \nabla_{1} u(s, y)\right|,
\end{aligned}
$$

respectively for $(x, y) \in Q$. Taking on both sides of $(14),(15),(16)$ the powers $p_{1}, p_{2}, p_{3}$ respectively and using the Hölder's inequality with indices $p_{1}$ and $\frac{p_{1}}{p_{1}-1}, p_{2}$ and $\frac{p_{2}}{p_{2}-1}$, $p_{3}$ and $\frac{p_{3}}{p_{3}-1}$ respectively on the right sides we get

$$
\begin{aligned}
|u(x, y)|^{p_{1}} & \leq\left(\frac{1}{2}\right)^{2 p_{1}}\{n m\}^{p_{1}-1} \sum_{s=1}^{n} \sum_{t=1}^{m}\left|\nabla_{2} \nabla_{1} u(s, t)\right|^{p_{1}}, \\
\left|\nabla_{1} u(x, y)\right|^{p_{2}} & \leq\left(\frac{1}{2}\right)^{p_{2}} m^{p_{2}-1} \sum_{t=1}^{m}\left|\nabla_{2} \nabla_{1} u(x, t)\right|^{p_{2}}, \\
\left|\nabla_{2} u(x, y)\right|^{p_{3}} & \leq\left(\frac{1}{2}\right)^{p_{3}} n^{p_{3}-1} \sum_{s=1}^{n}\left|\nabla_{2} \nabla_{1} u(s, y)\right|^{p_{3}}
\end{aligned}
$$

respectively. From (17)-(19) we observe that

$$
\begin{gathered}
|u(x, y)|^{p_{1}}\left|\nabla_{1} u(x, y)\right|^{p_{2}}\left|\nabla_{2} u(x, y)\right|^{p_{3}}\left|\nabla_{2} \nabla_{1} u(x, y)\right|^{p_{4}} \\
\leq L\left\{\sum_{s=1}^{n} \sum_{t=1}^{m}\left|\nabla_{2} \nabla_{1} u(s, t)\right|^{p_{1}}\right\}\left\{\sum_{t=1}^{m}\left|\nabla_{2} \nabla_{1} u(x, t)\right|^{p_{2}}\right\} \\
\cdot\left\{\sum_{s=1}^{n}\left|\nabla_{2} \nabla_{1} u(s, y)\right|^{p_{3}}\right\}\left|\nabla_{2} \nabla_{1} u(x, y)\right|^{p_{4}} .
\end{gathered}
$$

where $L$ is as defined in Theorem 3. Now taking the sum on both sides of (20) first from $y=1$ to $m$ and then from $x=1$ to $n$ and using Schwarz inequality repeatedly and 
rewriting the sums we observe that

$$
\begin{aligned}
& \sum_{x=1}^{n} \sum_{y=1}^{m}|u(x, y)|^{p_{1}}\left|\nabla_{1} u(x, y)\right|^{p_{2}}\left|\nabla_{2} u(x, y)\right|^{p_{3}}\left|\nabla_{2} \nabla_{1} u(x, y)\right|^{p_{4}} \\
& \leq L\left\{\sum_{s=1}^{n} \sum_{t=1}^{m}\left|\nabla_{2} \nabla_{1} u(s, t)\right|^{p_{1}}\right\} \cdot \sum_{x=1}^{n} \sum_{y=1}^{m}\left\{\sum_{t=1}^{m}\left|\nabla_{2} \nabla_{1} u(x, t)\right|^{p_{2}}\right\} \\
& \cdot\left\{\sum_{s=1}^{n}\left|\nabla_{2} \nabla_{1} u(s, y)\right|^{p_{3}}\right\} \cdot\left|\nabla_{2} \nabla_{1} u(x, y)\right|^{p_{1}} \\
& \leq L\left\{\sum_{s=1}^{n} \sum_{t=1}^{m}\left|\nabla_{2} \nabla_{1} u(s, t)\right|^{p_{1}}\right\} \\
& \cdot\left\{\sum_{x=1}^{n} \sum_{y=1}^{m}\left(\sum_{t=1}^{m}\left|\nabla_{2} \nabla_{1} u(x, t)\right|^{p_{2}}\right)^{2}\left(\sum_{s=1}^{n}\left|\nabla_{2} \nabla_{1} u(s, y)\right|^{p_{3}}\right)^{2}\right\}^{\frac{1}{2}} \\
& \cdot\left\{\sum_{x=1}^{n} \sum_{y=1}^{m}\left|\nabla_{2} \nabla_{1} u(x, y)\right|^{2 p_{1}}\right\}^{\frac{1}{2}} \\
& \leq L(n m)^{\frac{1}{2}}\left\{\sum_{x=1}^{n} \sum_{y=1}^{m}\left|\nabla_{2} \nabla_{1} u(x, y)\right|^{2 p_{1}}\right\}^{\frac{1}{2}} \cdot(n m)^{\frac{1}{2}}\left\{\sum_{x=1}^{n} \sum_{y=1}^{m}\left(\sum_{t=1}^{m}\left|\nabla_{2} \nabla_{1} u(x, t)\right|^{2 p_{2}}\right)\right. \\
& \left.\cdot\left(\sum_{s=1}^{n}\left|\nabla_{2} \nabla_{1} u(s, y)\right|^{2 p_{3}}\right)\right\}^{\frac{1}{2}} \cdot\left\{\sum_{x=1}^{n} \sum_{y=1}^{m}\left|\nabla_{2} \nabla_{1} u(x, y)\right|^{2 p_{4}}\right\}^{\frac{1}{2}} \\
& =K \prod_{i=1}^{4}\left\{\sum_{x=1}^{n} \sum_{y=1}^{m}\left|\nabla_{2} \nabla_{1} u(x, y)\right|^{2 p_{i}}\right\}^{\frac{1}{2}}
\end{aligned}
$$

This completes the proof of Theorem 1.

Taking $p_{1}=p_{2}=p_{3}=p_{4}=1$ in (2) and using the Schwarz inequality on the right side of the resulting inequality we get the required inequality in (3). This completes the proof of Theorem 2.

The proof of Theorem 3 follows by closely looking at the proof of Theorem 1 and the proof of Theorem 4 is immediate by taking $p_{1}=p_{2}=p_{3}=1 \mathrm{in}(4)$ and using the Hölder's inequality with indices 3 and $\frac{3}{2}$ on the right side of the resulting inequality.

\section{References}

[1] P. R. Beesack, "On certain discrete inequalities involving partial sums", Canadian Jour. Math. 21, 222-234, 1969.

[2] L. K. Hua, "On an inequality of Opial", Sci. Sinica 14, 789-790, 1965.

[3] C. M. Lee, "On a discrete amalogue of inequalities of Opial and Yang", Canadian Math. Bull. 11, 73-77, 1968.

[4] D. S. Mitrinović, "Analytic Inequalities", Springer-Verlag, Berlin, New York 1970.

[5] Z. Opial, "Sur une inegalite", Ann. Polon. Math. 8, 29-32, 1960. 
[6] B. G. Pachpatte, "On certain discrete inequalities in two independent variables", Soochow Jour. Math. 11, 91-95, 1985.

[7] B. G. Pachpaite, "A note on Opial and Wirtinger type discrete inequalities", J. Math. Anal. Appl. $127,470-474,1987$.

[8] J. S. W. Wong, "A discrete analogue of Opial's inequality", Canadian Math. Bull. 10, 115-118, 1967.

Department of Mathematics and Statistics, Marathwada University, Aurangabad 431004, (Maharashtra), India. 